\title{
Real Time Face Detection using CCTV Camera
}

\author{
Nirmal Kumar Saraswat, Arvinda Kushwaha
}

\begin{abstract}
Closed Circuit Television i.e. CCTV are widely for security purposes getting the opportunity to be useful with time. Human face identification is one of major interests for this technology. In this paper, Human face is detection method is proposed with better accuracy and speed. This method can find wide use in this technological era as biometric identification is one of the best method of verification. In the proposed method divulgence of different facial parts, such as, Nose, Eyes \& Mouth could be done effectively and rapidly, without being concerned of the light or illumination in the background of the person. For this we have used Ada Boost Algorithm through which quick and precise results have obtained that are far better than that of previous methods. The results presents critical improvement utilizing introduced technique over different past systems. It might be visible that proposed procedure is staggeringly able with basic spurring power in observation usage. This method find its extensive Human face detection capabilities for security purposes in identifying the person among the group of living or non-living objects.
\end{abstract}

Index Terms: Surveillance, Human Face recognition, Security, CCTV.

\section{INTRODUCTION}

Application of the CCTV for video monitoring has increased exponentially specially after the 9/11 airplane hijack attack in New York \& 26/11 attack in Mumbai. Unlimited cameras have been presented in open domains wherever around world, for example, strip shopping centers, medicinal facilities, universities, schools, transport terminals, railroad stations, plane terminals, banks \& vending machines. In any case, beginning at now there is no beneficial structure to absolutely use point of confinement of such gigantic CCTV course of action[1]. Mostly cameras framework depend upon human resource to visualize. This makes submitted observation inefficient and unfeasible [2]. The way that barrier powers just discovered exercises of fear based oppressors from put away chronicles after attacks shows that present reconnaissance systems, which depends on manual checking, are neither reliable nor promising [3, 4]. Henceforth, highly capable smart CCTV structure is the need of the hour. Human Face recognition is among major requirement for CCTV structure [5, 6]. Using smart CCTV technology face recognition is possible that filter out the POI from the human face image. Here, different areas of the face recognized \& various elements like; solid structures, tress \& bodies etc are rejected from the electronic picture. Face recognition plays an important role in the majority of the human recognition frameworks. Here, paper deals with 5 sections; section 2 represents a literature survey, section 3 explains the used algorithm, section 4 explains analysis \& results and section 5 finishes the paper.

Revised Manuscript Received on October 30, 2019.

* Correspondence Author

Nirmal kumar Saraswat*, Deptt. of Computer Science, HRIT, Ghaziabad.

Arvinda Kushwaha, Deptt. of Computer Science, HRIT, Ghaziabad.

(C) The Authors. Published by Blue Eyes Intelligence Engineering and Sciences Publication (BEIESP). This is an open access article under the CC BY-NC-ND license (http://creativecommons.org/licenses/by-nc-nd/4.0/)

\section{LITERATURE SURVEY}

Two methods are used to recognize the Facial areas in the given image as feature base \& image base. In Feature based method, the features of the image are focused such as contrasting \& characteristics of face features, while Image based method, focuses on to attain proper relation between the preparing \& test image.

\section{A. Feature based approaches}

I. Active Shape model:

In active shape model we focus non grid features[1,3]. This model take training from given features and characterize the spot and we did modeling on behalf of that parameter and discover the target using that features.

\section{Low Level analysis}

It is carried out on low dimension visual characteristics, for example, shading, power, movement and edges and so on.

\section{Feature Analysis}

Here, the algorithm filters the parts that contain background, solid structures and illumination conditions to obtain the human face. There are combinations of different methods that are used to evaluate the features. We also use image based approach contain SVM [4] and PCA [3] and neural network.

\section{PROPOSED ALGORITHM}

This paper is used to distinguish various facial zones with given image. The picture preparing approach should reproduce the info picture to various sizes and after that execute fixed-size identification via those images. This strategy is tedious process because of computations of various size images. In introduced methodology, scale invariant component is conceived built utilizing integral picture \& some essential rectangular highlights like Haar highlights [7].

\section{A. Scale Invariant Features}

First convert the image into integral image then alter the sum of all pixels the concern pixel as depicted below.

\begin{tabular}{|l|l|l|}
\hline 1 & 1 & 1 \\
\hline 1 & 1 & 1 \\
\hline 1 & 1 & 1 \\
\hline
\end{tabular}

Input image

\begin{tabular}{|l|l|l|}
\hline 1 & 2 & 3 \\
\hline 2 & 4 & 6 \\
\hline 3 & 6 & 9 \\
\hline
\end{tabular}

Integral image

\section{Fig.1: Integral image}

We summing all pixel that are inside the rectangular window that cover all the vertices of image. 


\section{Real Time Face Detection using CCTV Camera}

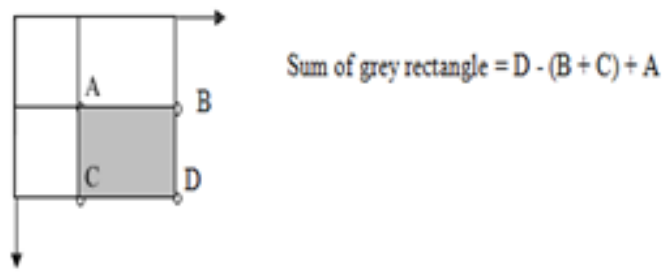

Fig. 2: Computation of Sum

\section{B. AdaBoost algorithm.}

In every feature, some highest points of the face are used to get the reliable information which enables the face recognition to be done efficiently. This can be done by slightly modifying the existing algorithm known as modified AdaBoost algorithm.

Ada boost algorithm is a machine learning algorithm. Use solid classifier based on weighted features is to be known as frail classifier.

$$
g(x, f, p, \theta)=\left\{\begin{array}{lr}
1 \text { if } p f(x)>p \theta \\
0 & \text { otherwise }
\end{array}\right.
$$

$\mathrm{p}$ is polarity and $\mathrm{f}$ is features and $\theta$ is threshold so we check that value of $x$ (positive or negative).

The introduced modified AdaBoost algorithm is shown in fig.3. it used best feature, polarity \& threshold. There is apparently no best answer for this issue \& introduced system prescribes a basic power method. It prescribes the guarantee of each latest frail classifier incorporates evaluating every part assuming every one of the cases keeping the ultimate objective to find the best performing feature.

\section{ALGORITHM FOR CLASSIFICATION}

The instruction in the algorithm measured pictures is sifted with the assistance of an identifier. Practically, a picture containing at least one human face can produce large no. of the surveyed in different -window. issue: as opposed to finding facial parts, the computation ought to discard nonfacial parts.

Using this it is bit easy to filter out the non-facial parts from an image containing human face parts. In context on this a locator including just a solitary (solid) classifier out of the blue radiates an impression of being inefficient since the appraisal time is consistent paying little heed to the data. Thus the prerequisite for a frail classifier rises. The idea is depicted underneath with two stages is shown fig. 4.

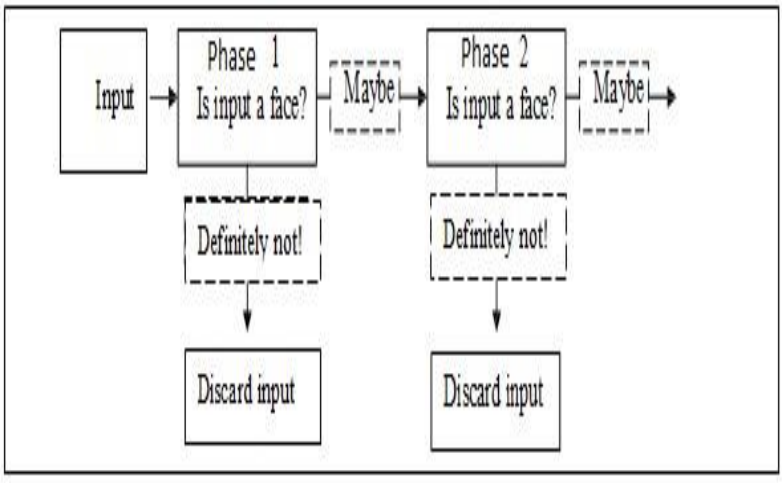

Fig. 4: Approach for classification

This paper informs about the position of image and locates the position and process the coordinate and negative outline tells about false negative at various stage and filter the results nearby.

\section{A. Sampling.}

The image is $102 * 1024$ that is adjusted into sub block of $24 * 24$



Fig. 5: Sample positive images

The change in the set is suggested as a mean of reducing the effect of different illuminations. Fig. 6 exhibits an example of standardized picture.

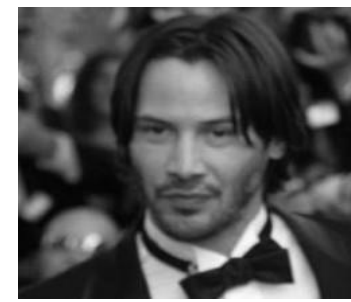

Fig. 6: Normalized image

\section{B. Creating Negative Samples}

A set of pictures that has non-facial parts are known as negative sample [6]. This way may at first, have every one of the reserves of being unquestionably not difficult to satisfy. Test negative pictures are appeared in Fig. 7. 
a) Given sample images $\left(x_{1}, y_{1}\right), \ldots \ldots \ldots . . .,\left(x_{n} y_{2} y_{n}\right)$ where $y_{i}=0$ for negative samples \& $y_{i}=1$ for positive samples.

b) Initialize weights $w_{1, i}=\frac{1}{2 l}$ for $y_{i}=0$ and w wil $=\frac{1}{2 l}$ for $y_{i}=1$ where $m$ is number of negatives samples \& L is number of positives samples.

c) For $t=1, \ldots \ldots \ldots . . ., T$ :

o Normalize weights

$$
w_{t, i} \approx \frac{w_{t i}}{\sum_{j=1}^{n} w_{t i j}}
$$

- Opt for best frail classifier in reference to weighted error.

$$
\mu_{t}=\min _{q, p, \theta} \sum_{i} w_{i}\left|g\left(x_{i j} f, p, \theta\right)-y_{i}\right|
$$

0 Define $g_{t}(x)=g\left(x, f_{t}, p_{t}, \theta_{t}\right)$ where $f_{t}, p_{t}$ and $\theta_{t}$ are minimisers of $\mu_{t}$.

0 Update weights:

$$
W_{t+1, i}=W_{t: j} \beta^{1-\theta_{i}}
$$

where $e_{i}=0$ if sample $x_{i}$ is classified correctly \& $e_{i}=1$ otherwise, \&

$$
\beta_{t}=\frac{\mu_{t}}{1-\mu_{t}}
$$

\section{d) Final solid or strong classifier is}

$$
\text { Classifier }(x)= \begin{cases}1 \text { if } \sum_{t=1}^{T} a_{t} g_{t}(x) \geq \sum_{2}^{1} \sum_{t=1}^{T} a_{t} \\ 0 & \text { otherwise }\end{cases}
$$

(6)

$$
\text { where } a_{t}=\log \frac{1}{\beta_{t}}
$$

Fig 3: The moditjed diddoostalgorithm.


Fig. 7: Sample negative images.

\section{Classifier Training}

In this we classify the image for positive and for every set like first and third relatively calling and organize, we use reinforced algorithm for false negative Ad boost algorithm not support the false negative. Methodology that use the classifier to reach the phase until we reach the target in positive model we use the key estimation of image.

\section{Visual Results}
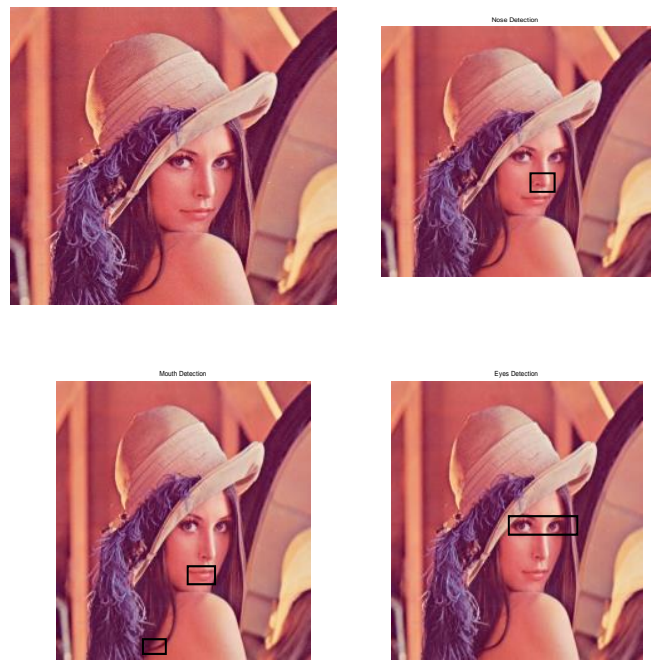

8 (a)
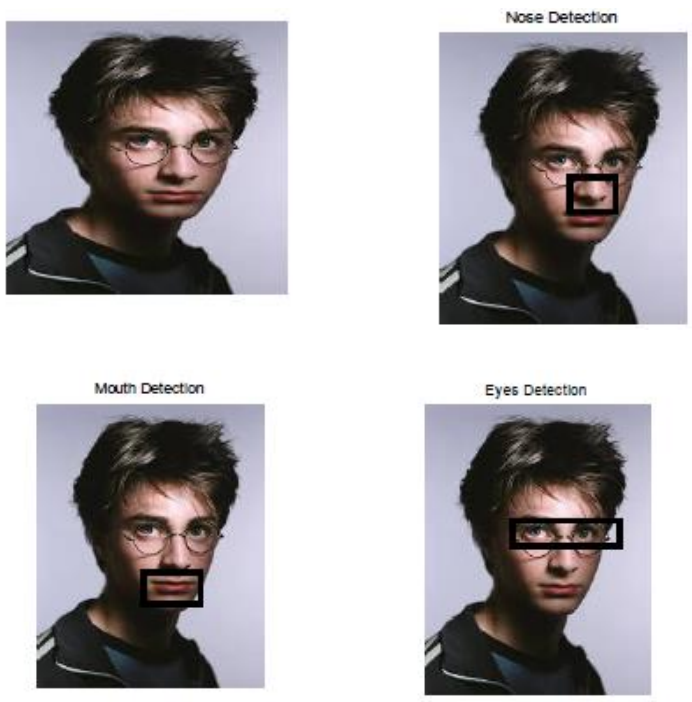

8(b) 


\section{Real Time Face Detection using CCTV Camera}
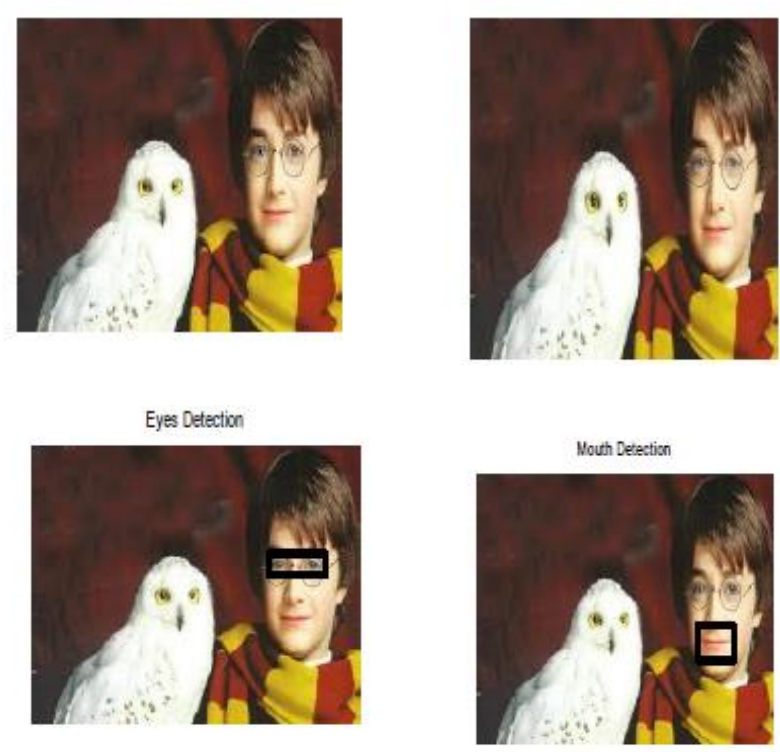

$8(c)$

Above image use different facial parts and recognized accurately

b part represent various illumination

c part image clarified the human eye and non human eye..

fig mention the change in brightening conditions, variety in face present, variety in outward appearances, variety in period of people and complex foundation. In any case, we get right recognition results for all the above difficulties.

Table 1: Experimental Results.

\begin{tabular}{|c|r|r|r|r|r|}
\hline Sr. No. & Images & \multicolumn{2}{|c|}{ Positive evaluation set } & \multicolumn{2}{c|}{ Negative evaluation set } \\
\cline { 3 - 6 } & & TPR & FNR & FPR & TNR \\
\hline 1 & 50 & 0.9740 & 0.0260 & 0.3815 & 0.6185 \\
\hline 2 & 100 & 0.9740 & 0.0260 & 0.4157 & 0.5843 \\
\hline 3 & 150 & 0.9740 & 0.0260 & 0.4919 & 0.5081 \\
\hline 4 & 200 & 0.9740 & 0.0260 & 0.4864 & 0.5136 \\
\hline 5 & 250 & 0.9740 & 0.0260 & 0.2272 & 0.7728 \\
\hline 6 & 300 & 0.9740 & 0.0260 & 0.3321 & 0.6679 \\
\hline 7 & 350 & 0.9740 & 0.0260 & 0.3970 & 0.6030 \\
\hline 8 & 400 & 0.9720 & 0.0280 & 0.3538 & 0.6462 \\
\hline 9 & 450 & 0.9700 & 0.0300 & 0.3943 & 0.6057 \\
\hline 10 & 500 & 0.9700 & 0.0300 & 0.4133 & 0.5867 \\
\hline 11 & 550 & 0.9680 & 0.0320 & 0.2804 & 0.7196 \\
\hline 12 & 600 & 0.9680 & 0.0320 & 0.3053 & 0.6947 \\
\hline 13 & 650 & 0.9680 & 0.0320 & 0.4333 & 0.5667 \\
\hline 14 & 700 & 0.9680 & 0.0320 & 0.4881 & 0.5119 \\
\hline 15 & 750 & 0.9680 & 0.0320 & 0.2366 & 0.7634 \\
\hline 16 & 800 & 0.9680 & 0.0320 & 0.4393 & 0.5607 \\
\hline 17 & 850 & 0.9730 & 0.0270 & 0.3548 & 0.6452 \\
\hline 18 & 900 & 0.9680 & 0.0320 & 0.3053 & 0.6947 \\
\hline 19 & 950 & 0.9740 & 0.0260 & 0.2272 & 0.7728 \\
\hline 20 & 1000 & 0.9720 & 0.0280 & 0.3538 & 0.6462 \\
\hline
\end{tabular}

\section{CONCLUSION}

I have shown various parts of face and accurately identified them using proposed method and use the concept of FPR and TPR, TRN, FNR. So, I develop a model that use training network and localize the object in very efficient manner.

Propose framework arrange the picture and figure out the parts even if the noise i:e buildings, animals, other humans etc are present in the image, the propose framework optimized the classification of image and disclose the structure.

It requires a lot of point by point tests tricky face recognizing verification pictures. These photographs unite faces under a broad accumulation condition, for example, scale, position, illumination and camera collection. The above proposed technique is amazing for face-detection as it provides efficient and effective results in much less time. Be that as it may, it might be reached out to different genuine applications like article identification, face location, and person verification, and so on for biometric acknowledgment in reconnaissance frameworks.

\section{REFERENCES}

1. Wu, Yu-Lung, \& Chi-Jui Chang. "Surveillance of public space: CCTV, privacy \& sense of safety." Global Journal for Research Analysis, vol. 5, no. 4 (2016).

2. Brands, Jelle, Tim Schwanen, \& Irina Van Aalst. "What are you looking at? Visitors' perspectives on CCTV in the night-time economy." European Urban and Regional Studies, vol. 23, no. 1 (2016): 23-39.

3. Sousa, William H., and Tamara D. Madensen. "Citizen acceptance of police interventions: an example of CCTV surveillance in Las Vegas, Nevada." Criminal Justice Studies, vol. 29, no. 1 (2016): 40-56.

4. Makin, David A., Gary Jenkins, \& Michael Gaffney. "Civilizing Surveillance Practices: The Pullman Police Department Public Safety Camera Monitoring Internship Program." Journal of Applied Security Research, vol. 11, no. 3 (2016): 249-266.

5. Wilber, Michael J., Vitaly Shmatikov, \& Serge Belongie. "Can we still avoid automatic face detection?" In 2016 IEEE Winter Conference on Applications of Computer Vision (WACV), pp. 1-9, 2016.

6. Yang, Shuo, Ping Luo, Chen-Change Loy, and Xiaoou Tang. "Wider face: A face detection benchmark." In Proceedings of IEEE Conference on Computer Vision and Pattern Recognition, pp. 55255533. 2016.

7. Umbaugh, Scott E. Digital image processing \& analysis: human \& computer vision applications with CVIPtools. CRC press, 2016.

\section{Authors Profile}

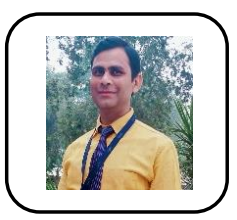

Nirmal Kumar Saraswat has completed his MCA from AKTU University Uttar Pradesh, India in 2009 and pursuing M.Tech (CSE) from HRIT Ghaziabad, Uttar Pradesh.

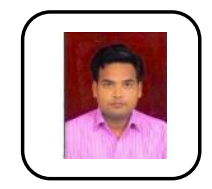

Arvinda Kushwaha received the B.Tech degree in CSE, from Bundelkhand University, Jhansi, U.P. in 2002 and M.Tech degree in Software Engineering from RGPV Bhopal, M.P. in 2012. Currently he is appearing Ph.D. degree in computer engineering from Jamia Millia Islamia, Delhi. More than 15 research papers has been published by him in international journals comprising Thomson Reuters (SCI \& Scopus) and conferences containing IEEE. Wireless sensor networks and cloud computing are his interested areas. 